\title{
Treatment of Oral Squamous Cell Carcinoma using Thymoquinone Loaded on Gold Nanoparticles
}

\author{
Merhan N. El-Mansy' ${ }^{*}$, Magda M. Hassan¹, Kholoud M. Abou El-Nour², \\ Wafaa H El-Hosary'.
}

${ }^{1}$ Department of ${ }^{1}$ Oral Pathology, Faculty of Dentistry, ${ }^{2}$ Department of Chemistry, Faculty of Science, Suez Canal University. Egypt

\begin{abstract}
Background: Oral cancer is one of the most prevalent cancers. Thymoquinone has a potential therapeutic activity against cancer cells. Aim: to compare the effect of thymoquinone and thymoquinone loaded on gold nanoparticles (GNPs-TQ) as systemic therapeutic agents through intraperitoneal injection for $3 \& 6$ weeks after painting of with DMBA 3 times/week for $14 \mathrm{w}$. Material and Methods: This study was carried out on ninety male Syrian golden hamsters $(n=90)$, its age ranged from 6 to 7 weeks, weighting 90-110 gm, purchased from Theodore Blhars Research Institute, Cairo, Egypt. Characterization of GNPs was achieved through using Ultraviolet-Visible spectroscope and transmission electron microscope (TEM). Tissue samples were examined through routine H\&E stain, and nuclear factor kappa B cells (NF-kB) immunoreactivity. Results: Formation of spherical and well dispersed gold nanoparticles (GNPs) (27nm). Histopathological evaluation showed retardation of carcinogenesis in hamster buccal pouch and regeneration of striated muscle layers when treated with thymoquinone loaded on gold nanoparticles (GNPs-TQ). These observations were in line with decrease nuclear factor-kappa B expression. Conclusions: GNPs-TQ is a promising chemo-therapeutic agent in regression of carcinogenesis, and improving the general animal health.
\end{abstract}

Key words: Thymoquinone, Gold nano-particles, HBP/DMBA, and NF-kB

\section{Introduction}

Oral cancer is the sixth most common cancer in the world(1). The overall 5-year survival rates represent $50 \%$ of population. It is directly related to the stage of diagno$\mathrm{sis}^{(2)}$. DMBA is a polycyclic aromatic hydrocarbon. It can mediate neoplastic transformation by inducing DNA damage through adduction to adenine and guanine residues in DNA, and generating excess reactive oxygen species(3). Syrian golden hamster is an excellent model for oral carcinogenesis that closely correlates with common events involved in the development of human oral cancers ${ }^{(4)}$. Thymoquinone is considered the main active constituent of black seed. Despite the promising outcomes in preclinical settings, nano-carriers were found to increase the efficacy, stability, solubility, and decreasing their toxicity(5). Therefore, TQ was studied in this formulation to achieve maximum benefits. Gold is known to be a noble metal. GNPs could be easily synthesized with different shapes as spheres, rods, tubes, or wires by 
changing reaction conditions. GNPs can be easily characterized due to presence of the characteristic surface Plasmon resonance (SPR) bands(5). Nuclear factor kappa B (NF$K B$ ) is responsible for regulating inflammation as well as the control of proliferation and apoptosis. It plays a role in cellular differentiation, survival and organogenesis ${ }^{(6)}$. This study was the first which aimed to evaluate the effect of thymoquinone loaded on gold nanoparticles, as a chemo-therapeutic agent in DMBA-induced hamster buccal pouch experimental model via intraperitoneal injection and detecting the immune reactivity of NF- $\kappa \mathrm{B}$.

\section{Material and Methods}

Chemicals: Tetra chloroauric acid, trisodium citrate to get GNPs solution. Thymoquinone and propylenegylcol to get thymoquinone solution. The chemical carcinogen 7,12 Dimethylbenz-[a]-anthracene, and heavy mineral oil to get DMBA solution. All the previous chemicals were purchased from Sigma Chemical Company, USA. NFkappa B/p65 antibody; (rabbit polyclonal antibody) was purchased from Thermo Fisher Scientific, Anatomical Pathology, UK to detect immunohistochemical expression.

Gold nano-particles preparation (GNPS) \& loading by TQ: All the steps were performed at room temperature. All the glassware's were rinsed with $10 \%$ nitric acid which kept clean and dry for use. Turkevich $\operatorname{method}^{(7)}$ was used to prepare GNPs. The gold nanoparticles were gradually formed as the trisodium citrate reduced the Au (III) to $\mathrm{Au}(\mathrm{O})$ as indicated by change in color from pale yellow to red color. A concentration of thymoquinone solution of $0.001 \mathrm{mg} /$ 100gm was prepared in propylene glycol by dissolving a certain amount of TQ crystals in propylene glycol through using a magnetic stirrer for 2 hours. Then, the solution of GNPs with thymoquinone was prepared by mixing equal amounts of both (1:1). The mixture was stirred on a magnetic stirrer for 2 hours and the formed solution was kept in $5^{\circ} \mathrm{C}$. Transmission electron microscope was used to determine the morphology of GNPs, GNPs-TQ by high resolution transmission electron microscope (TEM) using JEOL JEM 2100 (Japan). Pictures of nanoparticles were taken in the Egyptian Petroleum Research Institute. UltravioletVisible Spectroscope was used to determine the maximum absorption, GNPs, TQ, and GNP-TQ were studied by ultravioletVisible (UV-VIS) spectroscope that was recorded at room temperature with samples in a quartz cuvette using T90+UV-VIS Spectrometer, PG Instruments Ltd (wavelength range $250-850 \mathrm{~nm}$ ).

Experimental design: The chemical carcinogen was dissolved in heavy mineral oil to get $0.5 \%$ solution. It was topically applied to hamster left buccal pouches (HBP) by using number (4) camel hair brush. The experiment was held at the animal house in Pharmacology and Toxicology Department, Faculty of Pharmacy, Suez Canal University, Ismailia, Egypt.

Study groups: This study was carried out on ninety male hamsters $(n=90)$, weighting 90-110 gm, purchased from Tiedor Blhars Research Institute, Cairo, Egypt. Animals were housed, five per cage, and given pellets formed of seeds, grain, and tap water ad libitum. The hamsters were divided into: Group A: (Control groups): Thirty animals were divided into two subgroups; Subgroup A1: Twenty animals $(n=20)$ were served as "negative control" which didn't receive any treatment. Subgroup A2: Ten animals $(n=10)$ were considered as "positive control". DMBA was painted to the left cheek pouches, 3/week for 14 weeks. Group B: (self-control): Thirty animals were injected intra-peritoneally (i.p.) with GNPs, TQ, and GNPs-TQ 3 times/week for 3 and $6 \mathrm{w}$ respectively. Group C: (chemotherapeutic group) thirty animals were painted with DMBA to left cheek pouches 3 times/week 
for 14weeks, then the painting was stopped. The hamsters were treated systemically with intra-peritoneal injection of GNPs, TQ, and GNPs-TQ 3/week for 3 and $6 \mathrm{w}$ respectively.

Histopathological and immunohistochemical evaluation: After hamsters sacrificing, the pouches were surgically removed, fixed in $10 \%$ neutral formalin solution, processed, embedded in paraffin, and sectioned into $5 \mu \mathrm{m}$. sections were mounted on glass slides and stained with hematoxylin and eosin for light microscopic study, then photographed by E-330 Olympus digital camera. Other sections were mounted on positively-charged slides for immunohistochemical staining.

\section{Results}

Characterization of GNPs, and GNPs-TQ: The formation of GNPs was preliminarily confirmed by visual observation of color change from pale yellow to deep red color. TEM micrographs of GNPs showed spherical and well dispersed particles without agglomeration (Fig. 1-A). Most of particles were between 25-30 nm (Fig. 1-B).

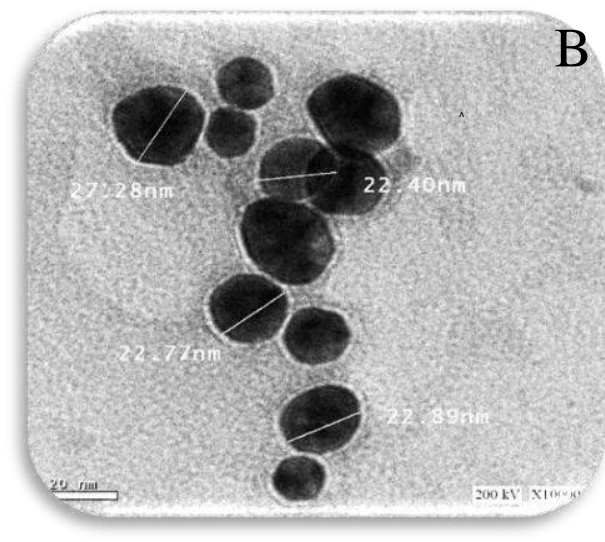

Figure 1: TEM micrographs of GNPs at magnification of 1:30.000 (A), 1:100.000 (B).

The produced solutions of GNPs, TQ, and GNPs-TQ were subjected to UV-visible spectroscopy. Sharp peak was given by UVvisible spectrum for GNPs at $\lambda \max =526 \mathrm{~nm}$ which confirmed the nanoparticles formation, the maximum absorption peak for TQ was recognized at $\lambda \max =316 \mathrm{~nm}$, while GNPs -TQ gave maximum absorption peak $\lambda \max =532 \mathrm{~nm}$ (Fig. 2). This deviation supported the loading of TQ on GNPs.

Clinical findings: The animals painted with DMBA for 14 weeks had skin ulcers or abscess with multiple exophytic masses. The pouch length was about $1.5 \mathrm{~cm}$ (Fig. 3). The same results were obtained in the groups treated with GNPs. Slight improvement was noted in animals treated with TQ for 6 wks. The groups treated with GNPs-TQ for
3 and 6 weeks showed marked improvement in general health of animals. There was a significant elongation of the pouches reached to about $3.5 \mathrm{~cm}$ after 6 wks. There was significant decrease in the size of exophytic masses rather than animals treated with GNPs or TQ only (Fig. 4). Histopathological and Immunohistochemical findings: Negative and self-control groups revealed normal hamster buccal pouch lining without any signs of dysplasia. Immuno-histochemical results revealed absence of NF-KB immune-reactivity (score $0)$. DMBA-painted animals for 14 weeks showed well differentiated scc in the form of large papillomatous lesion with deeply invading islands into underlying connective tissue with variable signs of dysplasia. 


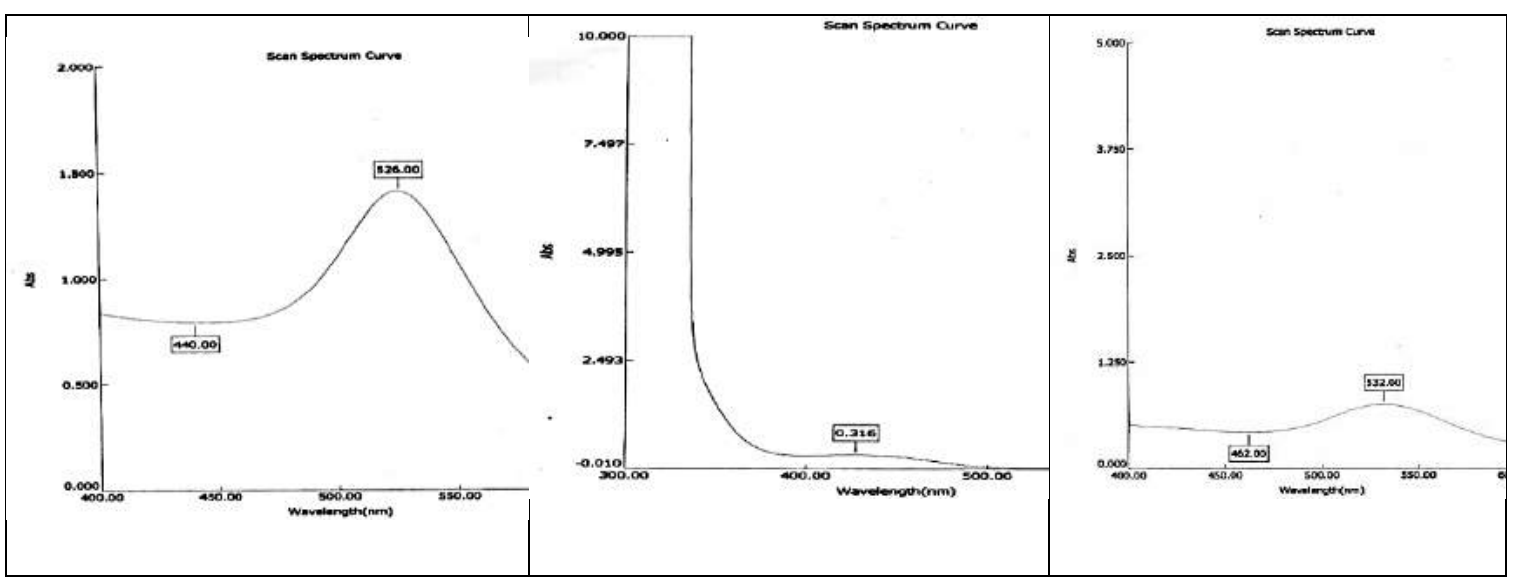

Figure 2: Photograph showing sharp peak of UV-visible spectrum for GNPs at $526 \mathrm{~nm}$ (right), for TQ at $316 \mathrm{~nm}$ (middle), and for GNPs-TQ at $532 \mathrm{~nm}$ (left).

The same results were obtained from both groups treated with GNPs for 3 and 6 w. The group treated with TQ for 3 w showed well differentiated scc in the form of nodular lesion with deeply invading islands of epithelium into underlying connective

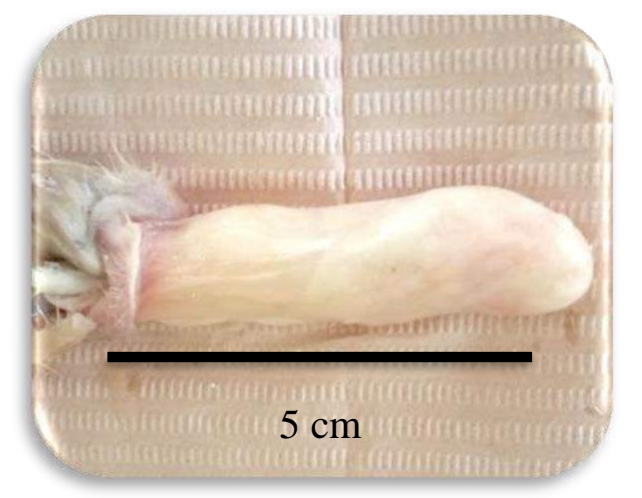

Figure 3: Photograph from group A showing normal hamster buccal pouch length (left). From group B showing protruding mass from the buccal pouch after with marked shortening of the pouch $(1.5 \mathrm{~cm})$.

The group treated with GNPs-TQ for 3 w revealed papillomatous lesion with superficial invasion which limited to the nodules only. This result was in cosistent with the group given TQ for $6 \mathrm{w}$ which indicated enhanced effect of the TQ after loading on GNPs. All the previous groups revealed intense nuclear immune reactivity of NF-kB (score 3). Significant improvement was no- tissue. The surface epithelium showed multiple signs of dysplasia. Extension the time of treatment to $6 \mathrm{w}$ showed superficial invasive scc which limited to nodule only, which verified regression of tumor.

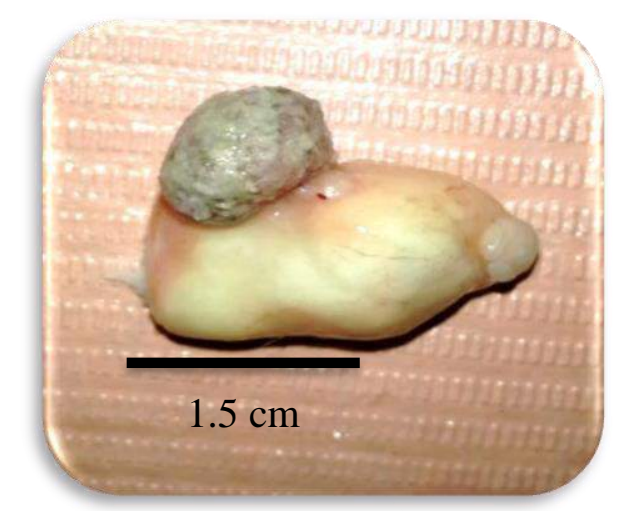

ted with extension the time of treatment to $6 \mathrm{w}$. There were only papillomatous lesions with no invasive islands. Epithelium showed variable signs of dysplasia from mild to carcinoma in situ. An important finding was seen as increase amount of the striated muscle layer. This group revealed moderate nuclear immune reactivity of NFkB (score 2) (Fig. 5 and 6). 
Figure 4: Photographs showing different sizes of tumor masses in group $C$ treated by (i.p) gold nanoparticles, thymoquinone, or gold nanoparticles loaded on thymoquinone after DMBA for 3 and 6 wks. (Note: length of the pouch in animal treated with GNPs-TQ for 6 weeks).
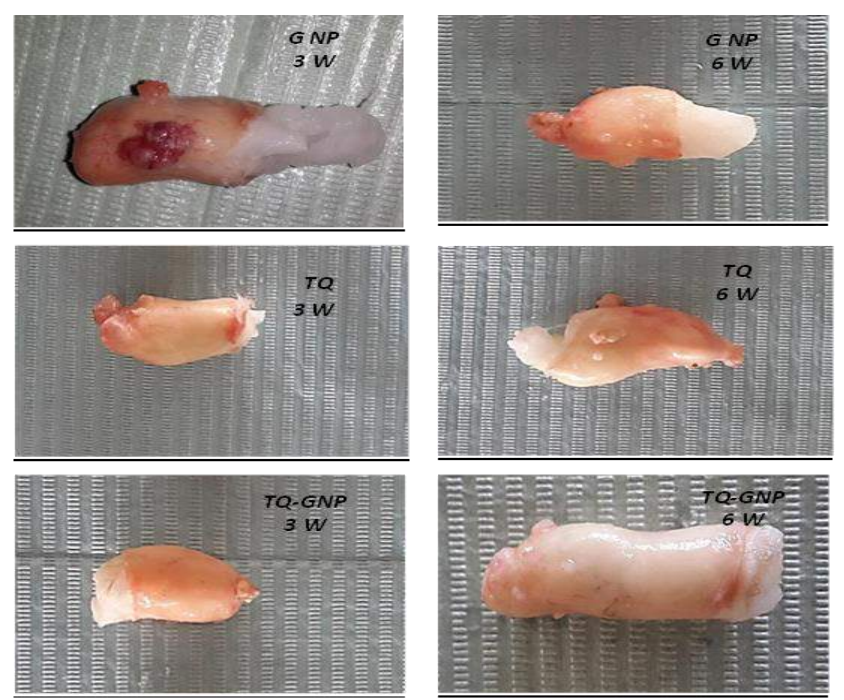

\section{Discussion}

This study was the first to investigate the effect of GNPs-TQ when given through intra-peritoneal injection on HBP/DMBA experimental model, and reporting elongation of the shortened cheek pouches with muscle regeneration. Tumor induction protocol of Shklar had been followed ${ }^{(8)}$. In this work, the technique used to prepare GNPs had resulted in spherical, well dispersed without agglomeration with the particle sizes between 25-30 nm. Pan et al (2007)(9) demonstrated that the cytotoxicity of GNPs depended on their size. The authors observed that GNPs of 1-2 nm in size were highly toxic, while particles larger than 15$\mathrm{nm}$ were comparatively nontoxic. The cellular uptake of spherical GNPs documented to be higher than rod-shaped counterparts ${ }^{(10)}$. Shortening of the DMBApainted pouches was recorded. Al Jawfi (2008) $)^{(11)}$ explained that, buccal necrosis may be due to either the local toxic effect of DMBA that elicited a severe inflammatory response, compared to untreated group. Of interest, by the end of intra-peritoneal injection of GNPs-TQ for 6 weeks, pouch elongation to about $3.5 \mathrm{~cm}$ was noted, as compared to the DMBA-painted group that was associated with regeneration of the striated muscle layer, seen histologically. This finding could confirm the better and direct effect on muscle regeneration of GNPs-TQ than TQ alone. An explanation of this finding, relating chronic inflammation and its effect on either muscle fibrosis or regeneration, is prevention of muscle fibrosis which improves muscle healing ${ }^{(12)}$. Furthermore, the histopathological findings in the present work confirmed a beam of light about the possible chemo-therapeutic effect of the used agents. Results in group B (DMBA 14W) showed well differentiated squamous cell carcinoma (SCC), with marked chronic inflammatory cells between invaded malignant epithelial islands. These findings were documented by several authors $(9,13)$. In the present work, chemotherapeutic effect through intra-peritoneal injection with TQ and GNPs-TQ resulted in delaying the malignancy process. The best results were obtained by using GNPs-TQ especially with extension the time of treatment that ends in carcinoma in situ. Also, TQ made delaying for the carcinogenesis process but in a lesser degree. The worst results were obtained with GNPs alone which indicated that it acted as a drug carrier for TQ and the aggressive behavior of cancer increased 
with extension time of application. It revealed that, using of a novel formulation of TQ enhanced its effect for better targeting of the cancer hallmarks in vitro, to increase its bioavailability, better distribution, as well as allow low amounts of the drug reaching target tumor ${ }^{(14,15)}$ Therefore, TQ was loaded on GNPs and used as chemotherapeutic in the present work.
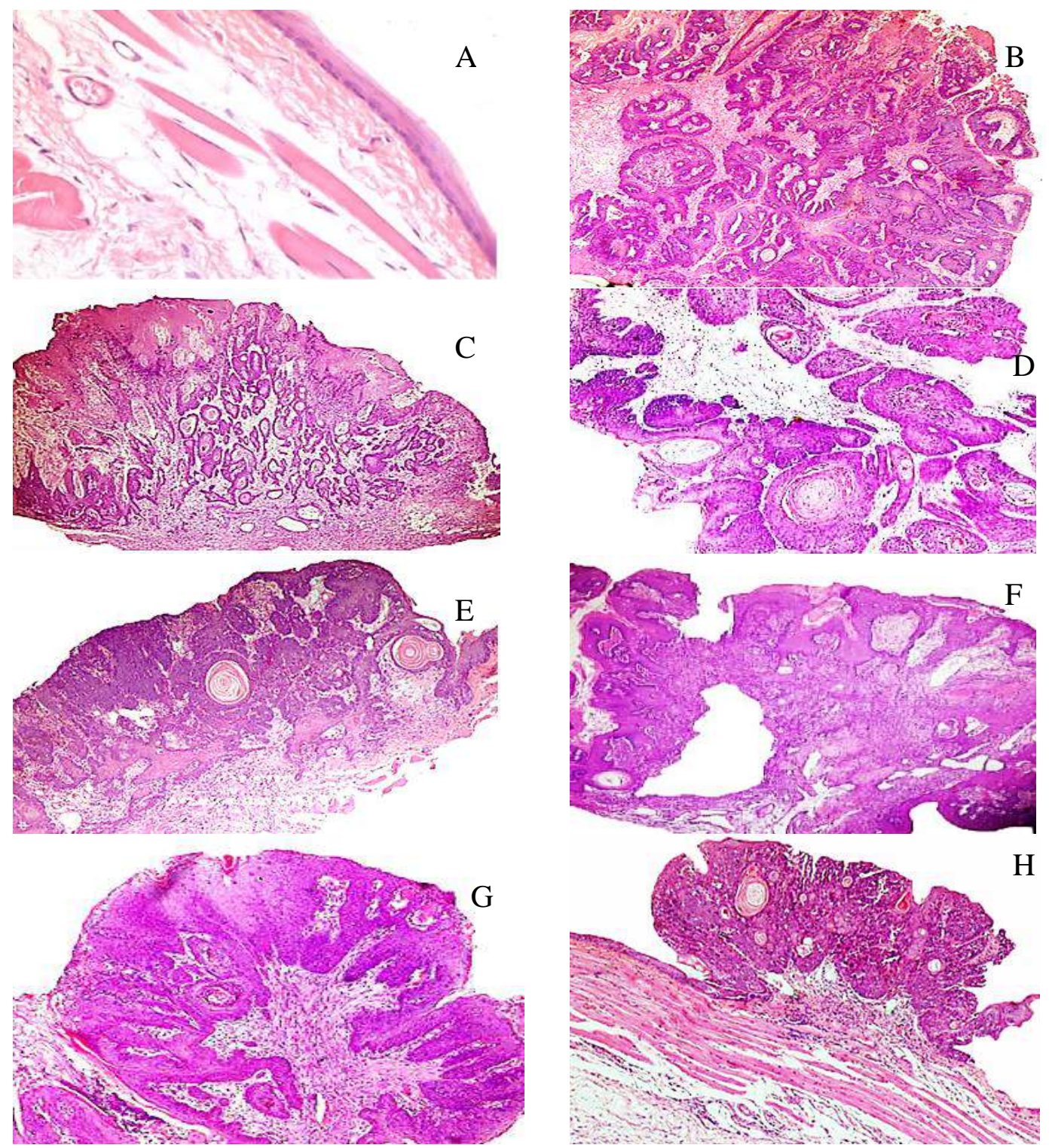

Figure 5: Histopathological photomicrographs (A) showing normal hamster buccal pouch X20, (B) well differentiated SCC, (C'D) well to moderate differentiated SCC, (E) deep invasion of well differentiated SCC, not limited within nodule, ( $\left.F^{\prime} G\right)$ superficial invasion of well differentiated SCC, limited within nodule, $(\mathrm{H})$ nodular lesion with moderate dysplasia to CIS with well-defined thickness of striated muscle layers (H\&E X4).

The present NF-KB immunohistochemical (IHC) findings of the present work confirmed the therapeutic effect of GNPs-TQ on the carcinogenesis process, most probably through its inactivation and suppres sion of chronic inflammatory response and induction of apoptosis. Regarding group $B$ (DMBA)-IHC result showed intense NF-kB reactivity (score 3 ) after 14 weeks. This result was online with Nagini et al ${ }^{(14)}$ who re 
vealed increase NF-kB expression with BP/DMBA model, for 14 weeks. In the present work, the best result was observed in group C6 (GNPs-TQ for 6 weeks) (score 2).
This finding would be explained due to painting of DMBA for long period which affected more oncogenes that controlled by the NF-kB,
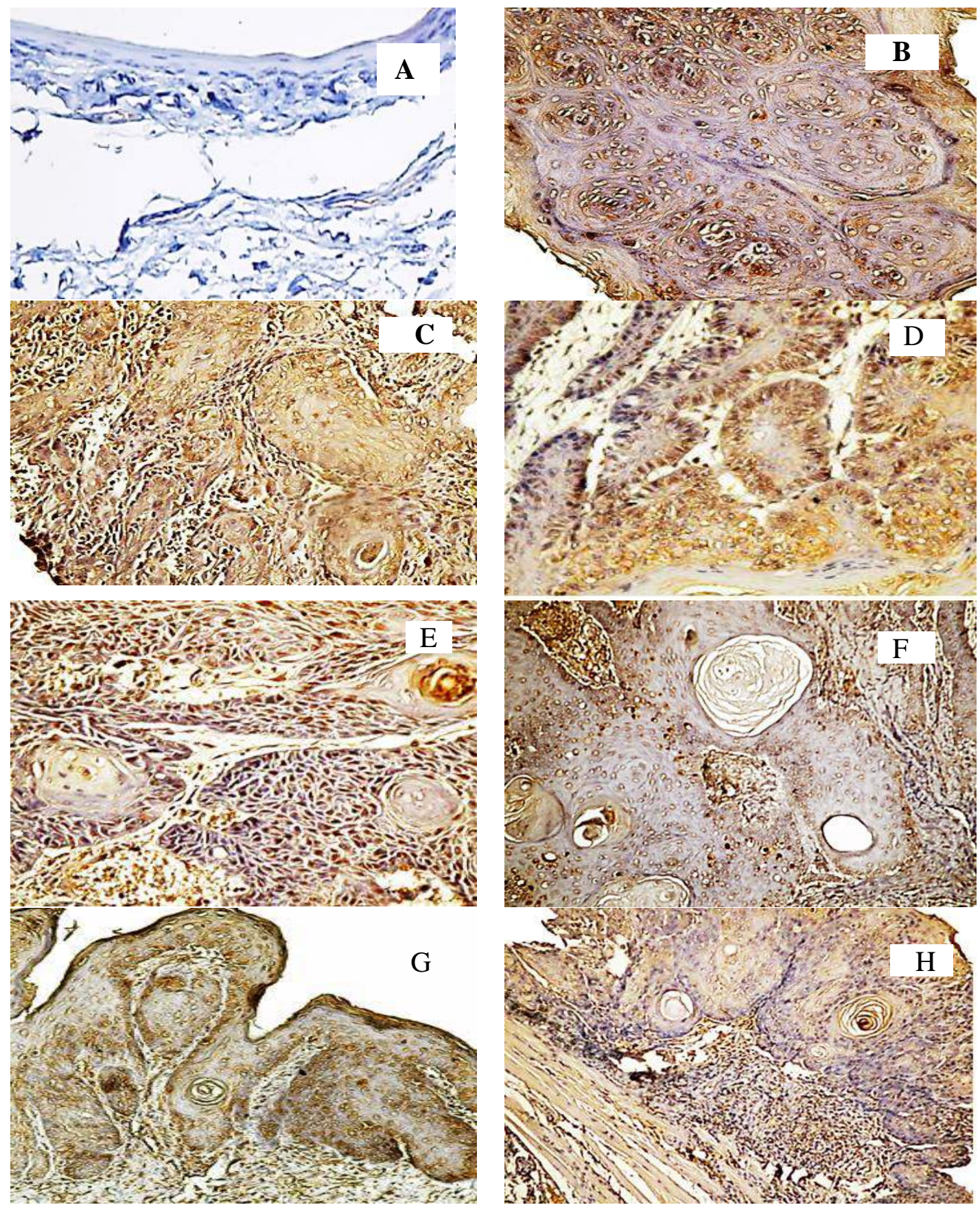

Figure 6: Immunohistochemical photomicrographs (A) with no immune-reactivity (score 0), (B-G) with intense nuclear immune-reactivity (score 3 ), $(\mathrm{H})$ with moderate nuclear immunereactivity (score 2 ). 
i.e. another mechanism might control the inflammation-carcinogenesis pathway. Results from previous studies revealed a high constitutive activation of NF-kB in oral cancer tissues and that its expression gradually increased with increase ed severity of lesions from oral potentially malignant to invasive carcinoma ${ }^{(16,17)}$. It appeared clearly that GNP-TQ formulation enhanced NF-kB inhibition capability than TQ alone. This result was noted in other TQ nano formulations. Ravindran et $a l^{(18)}$ loaded TQ with PLGA/PEG, and documented its enhanced effect than TQ alone in inhibiting NF-KB activation, and in suppressing the expression of cyclin D1, and matrix metalloproteinase (MMP-9).

\section{Conclusions}

Intra-peritoneal application of TQ loaded on GNPs at a concentration of (0.001 $\mathrm{mg} / \mathrm{kg}$ body weight) proved effective in delaying or regression the malignancy process, enhancing the general health of the animals, elongation of the mucosal striated muscle layer with non-toxic effect, along the experimental period (14 weeks). These effects are mostly mediated through inhibition of NF-kB expression.

\section{References}

1. Feller L, Lemmer J. Oral squamous cell carcinoma: epidemiology, clinical presentation and treatment. J Cancer Ther. 2012; 3:263-68.

2. Sankaranarayanan R, Dinshaw K, Nene $\mathrm{BM}$, et al. Cervical and oral cancer screening in India. J Med Screen. 2006; 13(1):35-8.

3. Bendaly J, Zhao S, Neale JR, et al. 2Amino-3,8-dimethylimidazo-[4,5-

f]quinoxaline-induced DNA adduct formation and mutagenesis in DNA repairdeficient Chinese hamster ovary cells expressing human cytochrome P4501 $\mathrm{A} 1$ and rapid or slow acetylator $\mathrm{N}$ - acetyltransferase 2.Cancer Epidemiol Biomarkers Prev. 2007;16(7): 1503-9.

4. Salley JJ. Experimental carcinogenesis in the cheek pouch of the Syrian hamster. J Dent Res. 1954 Apr;33(2):253-62.

5. Patra CR, Bhattacharya R, Mukhopadhyay $D$, Mukherjee P. Fabrication of gold nano-particles for targeted therapy in pancreatic cancer. Adv Drug Deliv Rev. 2010;62(3):346-61.

6. Tsurutani J, Castillo SS, Brognard J, et al. Tobacco components stimulate Akt-dependent proliferation and $\mathrm{NF}$ kappaB-dependent survival in lung cancer cells. Carcinogenesis. 2005;26(7) :1182-95.

7. Turkevich J, Stevenson P, Hillier J. A study of the nucleation and growth processes in the synthesis of colloidal gold. Discov Fara Soc. 1951; 11: 55-75.

8. Shklar G. Experimental oral pathology in the Syrian hamster. Prog Exp Tumor Res. 1972;16: 518-38.

9. Pan Y, Neuss S, Leifert A, et al. Size-dependent cytotoxicity of gold nanoparticles. Small.2007;3(11): 1941-9.

10. Chithrani BD, Ghazani AA, Chan WC.Determining the size and shape dependence of gold nanoparticles uptake into mammalian cells. Nano Lett. 2006; 6(4):662-8.

11. AL-Jawfi KAM, Hassan MMA, El-Gohary AM. Effect of Nigella sativa oil on the hamster lymphocytes secondary to DMBA-induced carcinogenesis. Suez Canal Univ Med J. 2008; 11(1):75-80.

12. Huard J, Li Y, Fu FH. Muscle Injuries and Repair: Current trends in research. J Bone Joint Surg Am. 2002; 84 (5): 822 -32 .

13. Nagini S, Letchoumy PV, A T, Cr R. Of humans and hamsters: a comparative evaluation of carcinogen activation, DNA damage, cell proliferation, apoptosis, invasion, and angiogenesis in oral cancer patients and hamster buccal pouch carcinomas. Oral Oncol.2009;45 (6): 31-7.

14. Schneider-Stock R, Fakhoury IH, Zaki AM, El-Baba CO, Gali-Muhtasib HU. Thymoquinone: fifty years of success in 
the battle against cancer models, Drug Discov Today. 2014; 19 (1):18-30.

15. Ganea GM, Fakayode SO, Losso JN, van Nostrum CF, Sabliov CM, Warner IM. Delivery of phytochemical thymoquinone using molecular micelle modified poly (D, L lactide-co-glycolide) (PLGA) nanoparticles. Nanotechnology, 2010; 21 (28): 285104.

16. Bindhu OS, Ramadas K, Sebastian P, Pillai MR. High expression levels of nuclear factor kappa B and gelatinases in the tumorigenesis of oral squamous cell carcinoma. Head Neck. 2006; 28 (10): 916-25.

17. Pontes HA, Pontes FS, Fonseca FP, et al. Nuclear factor- $\mathrm{kB}$ and cy-clooxygenase-2 immunoexpression in oral dysplasia and oral squamous cell carcinoma. Ann Diagn Pathol. 2013;17(1): 45-50.

18. Ravindran J, Nair HB, Sung B, Prasad S, Tekmal RR, Aggarwal BB. Thymoquinone poly (lactide-co-glycolide) nanoparticles exhibit enhanced anti-proliferative, anti-inflammatory, and chemosensitization potential. Biochem Pharmacol. 2010;79(11): 1640-7. 
